CASE REPORT

\title{
"Cutaneous-type" angiosarcoma arising in a mature cystic teratoma of the ovary
}

\author{
M A den Bakker, A C Ansink, P C Ewing-Graham
}

J Clin Pathol 2006;59:658-660. doi: 10.1136/jcp.2005.029751

Benign and malignant somatic tumours arising in mature cystic teratomas of the ovary are a rare but recognised phenomenon. Squamous cell carcinoma is the most common somatic malignancy arising in ovarian teratomas, although many other types of tumour have been described. An angiosarcoma with "cutaneous" type typical features arising in a dermoid cyst of the ovary is reported. Vascular tumours have only rarely been described as secondary somatic tumours in ovarian teratomas. The diagnostic features and complications of such tumours are described.

$\mathrm{S}$ omatic tumours arising in mature cystic teratomas (MCTs; dermoid cysts) are uncommon. Malignant degeneration occurs in up to $2 \%$ of MCTs, with squamous cell carcinoma being the most commonly encountered tumour type. ${ }^{1-3}$ Various non-epithelial malignancies, however, have also been documented, most commonly as a mesenchymal component of a carcinosarcoma. ${ }^{4-7}$ Benign and malignant vascular tumours have only rarely been described as occurring in the ovary. ${ }^{58}$ Primary ovarian angiosarcoma is very rare, with less than 25 reported cases. ${ }^{5}$ Angiosarcoma arising as a somatic malignancy in MCT is rare, with only two cases reported in the literature. ${ }^{8}$ We report a third case of angiosarcoma ex-dermoid cyst of the ovary. The angiosarcoma partly showed the typical aspect of an angiosarcoma arising in skin and merging with a high-grade component similar to the soft tissue counterpart. The cells had clearly identifiable cell borders and were arranged in short whorling fascicles. In less cellular areas at the periphery of the tumour, irregular slit-like vascular structures were evident (fig 1A). Strong CD31 and factor-VIII-related antigen positivity (fig 1B) were observed after immunohistochemical analysis.

\section{CASE REPORT}

A 30-year-old woman presented with abdominal discomfort of several months' duration. Ultrasonographical examination showed a large mass in the lower abdomen. An explorative laparotomy showed a large, partly cystic tumour attached to the left ovary; the tumour was removed and a staging procedure with subtotal omentectomy was carried out. An initial evaluation of the specimen yielded a diagnosis of a high-grade spindle cell tumour, probably squamous cell carcinoma, with the tumour present in the omentum and in the biopsies of the peritoneum. After four courses of chemotherapy, debulking was carried out with the removal of the uterus, right ovary and residual omentum. Clinically, there was a favourable response to the chemotherapy, with minor residual tumour in the abdomen. A month after debulking, the patient was readmitted with severe nausea and vomiting. Imaging showed liver metastases and enlargement of retroperitoneal and mediastinal lymph nodes, multiple peritoneal tumour nodules and a large pelvic mass with entrapped small-bowel and large-bowel loops. Curative treatment was not possible and optimal palliative care was provided. The patient died 9 months after initial presentation; postmortem examination was not carried out.

Tissue from the initial laparotomy and the interval debulking was processed according to standard protocols. Immunohistochemical tests with antibodies to cytokeratin (AEl/AE3, NCL5D3 and KL1), vimentin, epithelial marker antigen, CD34 (QBEND10), CD31 (JC70a), factor-VIII-related antigen, placental-like alkaline phosphatase, $\alpha$-fetoprotein and $\beta$-human chorionic gonadothropin were carried out, with appropriate controls, in an automated immunostainer, using a polymer-based system (DAKO Envision, DAKO, Glostrup, Denmark). Tumour tissue samples retrieved from the paraffin wax blocks of the initial laparotomy were processed for electron microscopy.

Microscopy of the tumour tissue removed during the debulking procedure after chemotherapy showed a cellular tumour predominantly composed of spindle cells with irregular oval and elongated nuclei and coarse chromatin and distinct nucleoli. CD34 staining, however, was completely negative, as were the assays for cytokeratin, epithelial marker antigen and germ cell markers (placental-like alkaline phosphatase, $\alpha$-fetoprotein and $\beta$-human chorionic gonadotropin).

In contrast, slides from the tumour removed in the exploratory laparotomy showed a highly cellular spindle cell tumour arranged in long sweeping fascicles, devoid of slitlike vascular structures (fig 2).

Initial immunohistochemical staining proved inconclusive. Epithelial markers (cytokeratins and epithelial marker antigen) were negative, as was CD34. CD31 and factor-VIII-related antigen were not included in the panel at this time. Electron microscopy showed the presence of occasional poorly formed desmosomes, leading to an initial diagnosis of sarcomatoid squamous cell carcinoma. A review of the slides and extended immunohistochemical analysis, however, showed the endothelial nature of the tumour. Staining results were identical to those of the debulking material, with strong consistent CD31 staining and focal factor-VIII-related antigen staining. Slides from the cystic areas showed a typical mature cystic teratoma with keratinising squamous epithelium and associated skinadnexal structures. Within the areas with epidermal and dermal tissue, ramifying ectatic vascular structures were observed, lined by atypical endothelial cells with hyperchromatic nuclei (fig 3). The appearance of this component was identical to that of angiosarcoma arising in skin. These foci merged into solid areas of the sarcomatous component.

\section{DISCUSSION}

The occurrence of a malignant somatic tumour in the dermoid cysts of the ovary is a rare complication and is seen in approximately $1.4-2 \%$ of MCT. $^{1-3}$ Squamous cell carcinoma arising in MCTs is the most common tumour, constituting

Abbreviation: $\mathrm{MCT}$, mature cystic teratoma 

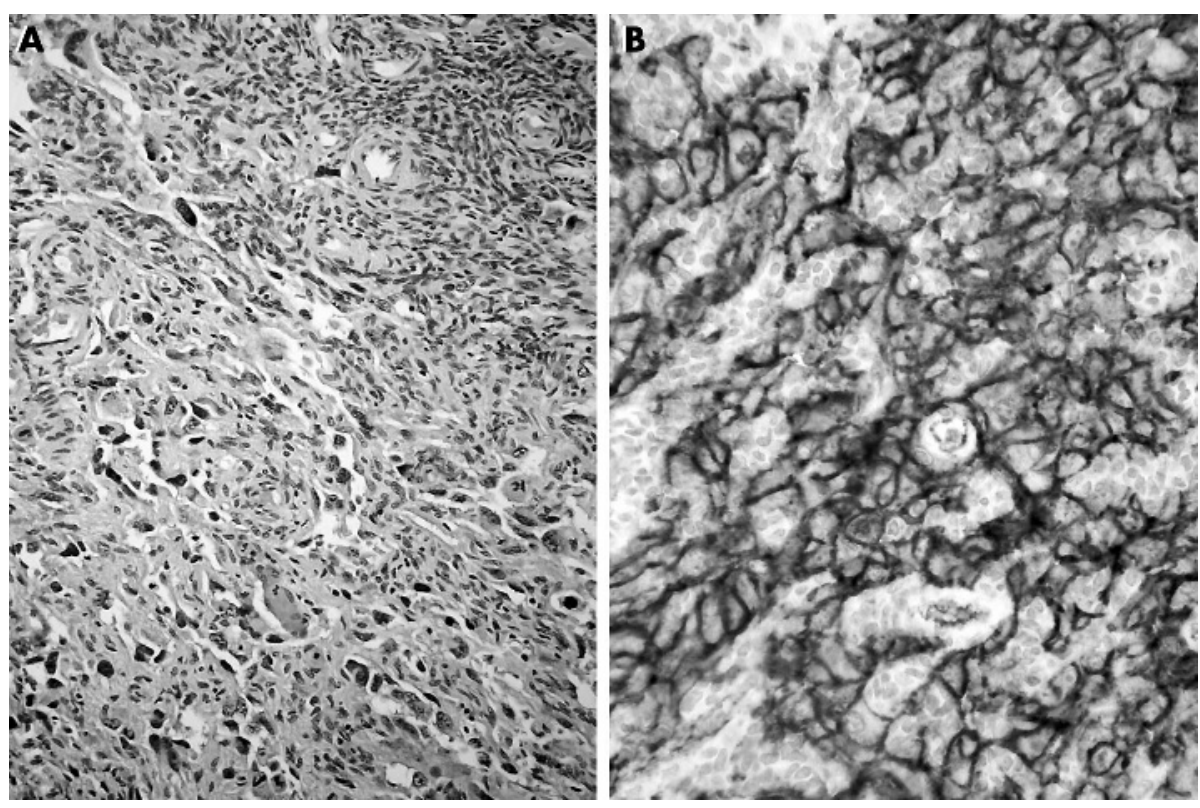

Figure 1 Tumour tissue from the debulking procedure. (A) Vague vascular architecture is discernible. (B) CD31 stain showing strong membranous positivity.

about $80 \%$ of secondary somatic malignancies in this setting. ${ }^{3}$ This is not surprising as the predominant tissue seen in MCTs is usually epidermal squamous epithelium with adnexa. Many other different types of benign and malignant somatic tumours have been described in MCTs, however, including melanocytic naevi and melanoma, skin-adnexal tumours, adenocarcinoma, carcinoids, carcinosarcoma and various other sarcomas (eg, osteosarcoma, rhabdomyosarcoma, fibrosarcoma, leiomyosarcoma and peripheral neuroectodermal tumour)..$^{4-689}$ These secondary tumours are similar to those seen in the sporadic setting and must be set apart from the malignant tissue in immature teratomas, which is at least partly composed of immature embryonic-type or fetal-type cells. The behaviour of somatic malignancies in MCT is not determined by their derivation from germ cells but by their phenotype, and thus is similar to that of their sporadic counterparts. ${ }^{6}$ Hence, treatment should be geared towards the somatic malignancy rather than towards a germ cell lesion.

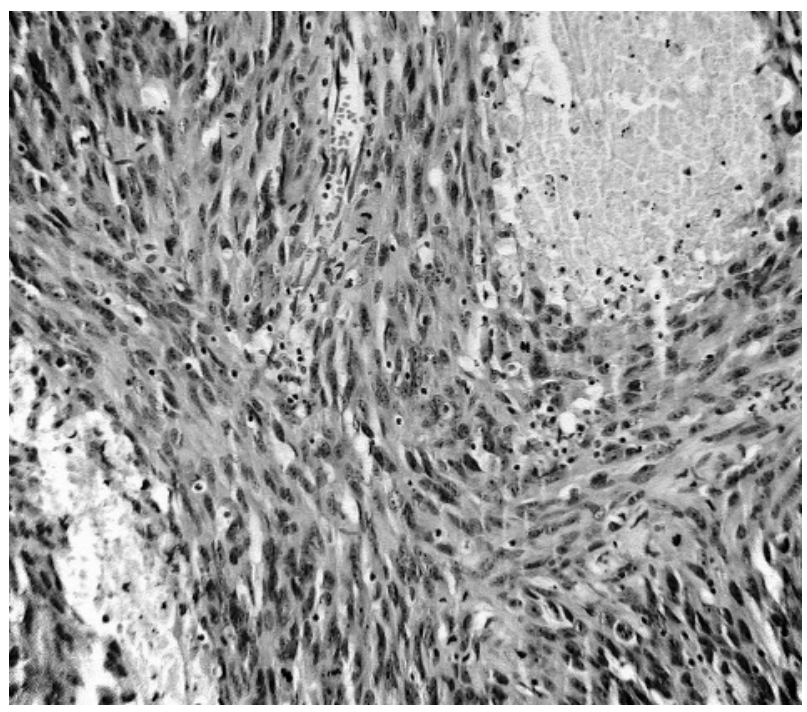

Figure 2 Tissue from the initial procedure showing a high-grade spindle cell tumour devoid of differentiation features.
Vascular tumours arising in MCTs of the ovary have only been described sporadically. ${ }^{5}$ Subtypes of haemangioma have been reported, including a mitotically active epithelioid vascular tumour that behaved in a benign fashion. ${ }^{810} 11$ Baker and Rosai described florid benign vascular proliferations in ovarian teratomas (MCT and immature teratoma) that may be confused with vascular neoplasms. These vascular proliferations were seen in teratomas with an extensive neural component and may have been induced by growth factors liberated by the neural component. ${ }^{12}{ }^{13}$

Malignant vascular tumours in the ovary are rare. No more than 25 primary ovarian angiosarcomas have been described to date, although the validity of a considerable number of these cases has been questioned. ${ }^{5}{ }^{14}$ In a number of cases, the angiosarcoma was seen in combination with an ovarian surface epithelial tumour. ${ }^{15}{ }^{16}$ Of the seven cases of primary ovarian angiosarcoma reviewed by Nielsen et al, ${ }^{8}$ two arose from dermoid cysts. Platt et $a l^{17}$ report a case of disseminated primary ovarian angiosarcoma, which was associated with two MCTs. The bulk of the angiosarcoma, however, was found in the ovary

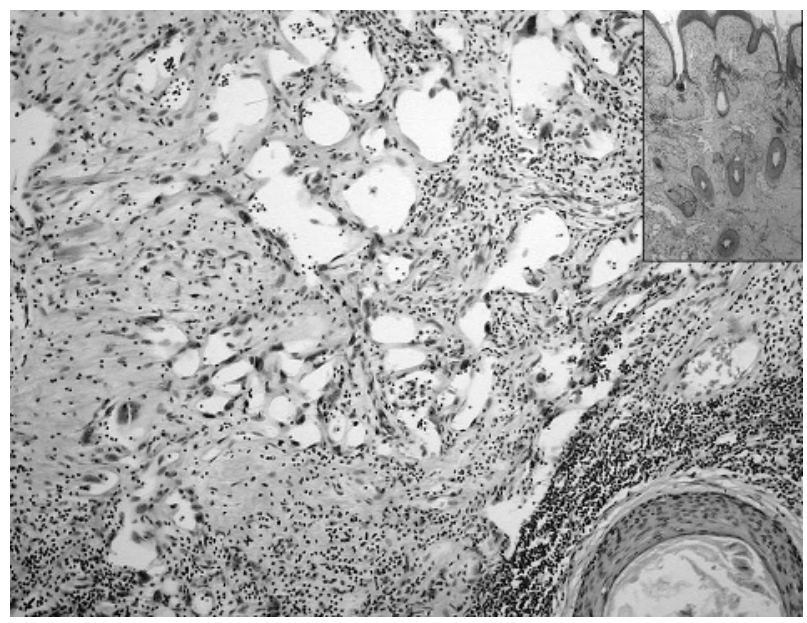

Figure 3 Tissue from the initial procedure showing a residual typical dermoid cyst structure. Atypical ectatic vascular structures are seen. Inset: low-power view with skin and adnexal structures of the dermoid cyst. 


\section{Take-home messages}

- Malignant degeneration occurs in up to $2 \%$ of ovarian mature cystic teratomas

- A great diversity of epithelial and mesenchymal secondary tumours have been described complicating mature cystic teratomas of the ovary

- Treatment of secondary non-germcell tumours in mature cystic teratomas should target the phenotype of the somatic tumour component rather than its germcell derivation

- CD31 is a sensitive and specific endothelial marker, which should be included in a panel of endothelial markers to confirm the diagnosis of a vascular tumour

contralateral to the one with the teratomas, which was described as being normal in size. It therefore seems unlikely that this is a third angiosarcoma ex-dermoid cyst but rather a primary ovarian angiosarcoma in combination with MCT. To the best of our knowledge, there are no other reported cases of angiosarcoma arising in MCTs in the literature in English. Incidental case reports do exist, however, of angiosarcoma complicating germ cell tumours in other locations..$^{19} 19$

The angiosarcoma described here partly showed typical features of angiosarcoma arising in skin, with irregular ectatic vascular structures ramifying through the dermal collagen. In contrast, (deep) soft-tissue and organ-based angiosarcoma usually shows features of a high-grade sarcoma and often requires immunohistochemical analysis to uncover its endothelial derivation. Whether these histological forms of angiosarcoma indeed represent different types is unclear. On the basis of the case presented here, in which cutaneous-type angiosarcoma merged with a high-grade spindled component, morphologically similar to the soft tissue equivalent of angiosarcoma, it could be argued that the high-grade soft-tissue type of angiosarcoma is no more than progression. The question remains of why angiosarcoma seems to have a predilection for cutaneous sites.

The initial diagnosis proved problematic, as the tumour consisted solely of a compact sarcomatous cell population arranged in long fascicles. Moreover, immunohistochemical analysis failed to show differentiation characteristics. Initially, CD34 had been used as the only endothelial marker and proved to be consistently negative. CD31 staining was performed only after morphological features in tissue from the debulking procedure suggested vascular differentiation, and was strongly positive. The superiority of CD31 in terms of sensitivity and, in particular, specificity for endothelial cells is well recognised and should ideally always be included in a panel in which an endothelial neoplasm enters the differential diagnosis. ${ }^{50-24}$

Electron microscopy showed occasional poorly formed desmosomes, leading to the diagnosis of squamous cell carcinoma. In retrospect, the demosomes may have been part of the squamous epithelium of the dermoid cyst or, more likely, were mistaken for adherens junctions, which may be seen in endothelial cells. ${ }^{25}$ Although cytokeratin staining was negative in this case, positive staining could have further complicated matters, as deep-seated angiosarcomas are frequently cytokeratin positive. ${ }^{52627}$

We conclude that the case presented here constitutes the third bona fide case of angiosarcoma ex-dermoid cyst of the ovary. In addition, this case illustrates the general fact that when faced with a morphologically undifferentiated tumour, supplemental techniques should be applied and interpreted with care.

\section{ACKNOWLEDGEMENTS}

We thank Dr J Boldewijn, St Clara Hospital, Rotterdam, for providing samples from the initial procedure.

\section{Authors' affiliations}

M A den Bakker, P C Ewing-Graham, Department of Pathology, Erasmus MC, Rotterdam, The Netherlands

A C Ansink, Departments of Obstetrics and Gynaecology, Erasmus MC, Rotterdam, The Netherlands

Correspondence to: Michael A den Bakker, Department of Pathology, Erasmus MC, PO Box 1738, 3000 DR Rotterdam, The Netherlands; m.denbakker@erasmusmc.nl

Accepted for publication 15 June 2005

\section{REFERENCES}

1 Ayhan A, Bukulmez O, Genc C, et al. Mature cystic teratomas of the ovary: case series from one institution over 34 years. Eur J Obstet Gynecol Reprod Biol 2000;88:153-7.

2 Zorlu CG, Kuscu E, Soysal ME, et al. Malignant degeneration of mature cystic teratomas. Aust N Z J Obstet Gynaecol 1996;36:221-2.

3 Peterson W. Malignant degeneration of benign cystic teratomas of the ovary: a collective reveiw of the literature. Obstet Gynecol 1957;12:793-830.

4 DiSaia PJ, Pecorelli S. Gynecological sarcomas. Semin Surg Oncol 1994; 10:369-73.

5 Nucci MR, Krausz T, Lifschitz-Mercer B, et al. Angiosarcoma of the ovary: clinicopathologic and immunohistochemical analysis of four cases with a broad morphologic spectrum. Am J Surg Pathol 1998;22:620-30.

6 Rosai J. Rosai and Ackerman's surgical pathology. Edinburgh: Mosby, 2004.

7 Azoury RS, Woodruff JD. Primary ovarian sarcomas. Report of 43 cases from the Emil Novak Ovarian Tumor Registry. Obstet Gynecol 1971;37:920-41.

8 Nielsen GP, Young RH, Prat J, et al. Primary angiosarcoma of the ovary: a report of seven cases and review of the literature. Int $J$ Gynecol Pathol 1997; 16:378-82.

9 Ribeiro-Silva A, Chang D, Bisson FW, et al. Clinicopathological and immunohistochemical features of a sebaceous carcinoma arising within a benign dermoid cyst of the ovary. Virchows Arch 2003;443:574-8.

10 Alvarez M, Cerezo L. Ovarian cavernous hemangioma. Arch Pathol Lab Med 1986;110:77-8.

11 Madison JF, Cooper PH. A histiocytoid (epithelioid) vascular tumor of the ovary: occurrence within a benign cystic teratoma. Mod Pathol 1989;2:55-8.

12 Baker PM, Rosai J, Young RH. Ovarian teratomas with florid benign vascular proliferation: a distinctive finding associated with the neural component of teratomas that may be confused with a vascular neoplasm. Int J Gynecol Pathol 2002;21:16-21.

13 Gaudin PB, Rosai J. Florid vascular proliferation associated with neural and neuroendocrine neoplasms. A diagnostic clue and potential piffall. Am J Surg Pathol 1995; 19:642-52.

14 Davidson B, Abeler VM. Primary ovarian angiosarcoma presenting as malignant cells in ascites: case report and review of the literature. Diagn Cytopathol 2005;32:307-9

15 Jylling AM, Jorgensen L, Holund B. Mucinous cystadenocarcinoma in combination with hemangiosarcoma in the ovary. Pathol Oncol Res 1999:5:318-9.

16 Pillay K, Essa AS, Chetty R. Borderline serous cystadenocarcinoma with coexistent angiosarcoma: an unusual form of ovarian carcinosarcoma. Int J Surg Pathol 2001;9:317-21.

17 Platt JS, Rogers SJ, Flynn EA, et al. Primary angiosarcoma of the ovary: a case report and review of the literature. Gynecol Oncol 1999;73:443-6.

18 Ulbright TM, Clark SA, Einhorn LH. Angiosarcoma associated with germ cell tumors. Hum Pathol 1985;16:268-72.

19 Manivel C, Wick MR, Abenoza P, et al. The occurrence of sarcomatous components in primary mediastinal germ cell tumors. Am J Surg Pathol 1986;10:711-7

20 DeYoung BR, Swanson PE, Argenyi ZB, et al. CD31 immunoreactivity in mesenchymal neoplasms of the skin and subcutis: report of 145 cases and review of putative immunohistologic markers of endothelial differentiation. J Cutan Pathol 1995:22:215-22.

21 Suster S. Recent advances in the application of immunohistochemical markers for the diagnosis of soft tissue tumors. Semin Diagn Pathol 2000; 17:225-35

22 Higgins JP, Montgomery K, Wang L, et al. Expression of FKBP1 2 in benign and malignant vascular endothelium: an immunohistochemical study on conventional sections and tissue microarrays. Am J Surg Pathol 2003;27:58-64.

23 Miettinen M. Immunohistochemistry in tumour diagnosis. Ann Med 1993;25:221-33.

24 Parums DV, Cordell JL, Micklem K, et al. JC70: a new monoclonal antibody that detects vascular endothelium associated antigen on routinely processed tissue sections. J Clin Pathol 1990;43:752-7.

25 Pavelka M, Roth J. Functional ultrastructure. Atlas of tissue biology and pathology. New York: Springer, 2005.

26 Meis-Kindblom JM, Kindblom LG. Angiosarcoma of soft tissue: a study of 80 cases. Am J Surg Pathol 1998;22:683-97.

27 Fletcher CD, Beham A, Bekir S, et al. Epithelioid angiosarcoma of deep soft tissue: a distinctive tumor readily mistaken for an epithelial neoplasm. Am J Surg Pathol 1991;15:915-24. 